\title{
PERMUTABILITY DEGREES OF FINITE GROUPS
}

\author{
D.E. OTERA ${ }^{\dagger}$ AND F.G. RUSSO $^{\dagger \dagger}$
}

Abstract. Given a finite group $G$, we introduce the permutability degree of $G$, as

$$
p d(G)=\frac{1}{|G||\mathcal{L}(G)|} \sum_{X \in \mathcal{L}(G)}\left|P_{G}(X)\right|,
$$

where $\mathcal{L}(G)$ is the subgroup lattice of $G$ and $P_{G}(X)$ the permutizer of the subgroup $X$ in $G$, that is, the subgroup generated by all cyclic subgroups of $G$ that permute with $X \in \mathcal{L}(G)$. The number $p d(G)$ allows us to find some structural restrictions on $G$. Successively, we investigate the relations between $p d(G)$, the probability of commuting subgroups $\operatorname{sd}(G)$ of $G$ and the probability of commuting elements $d(G)$ of $G$. Proving some inequalities between $p d(G)$, $s d(G)$ and $d(G)$, we correlate these notions.

\section{INTRODUCTION}

All the groups of the present paper are supposed to be finite. Given a group $G$ and its subgroup lattice $\mathcal{L}(G)$, the subgroup commutativity degree

$$
s d(G)=\frac{|\{(H, K) \in \mathcal{L}(G) \times \mathcal{L}(G) \mid H K=K H\}|}{|\mathcal{L}(G)|^{2}}
$$

of $G$ and the commutativity degree

$$
d(G)=\frac{|\{(x, y) \in G \times G \mid x y=y x\}|}{|G|^{2}}
$$

of $G$ have been largely studied in the last years. Fundamental properties and interesting generalizations of $s d(G)$ can be found in [11, 12, 13, 22, 27, 28, 29, and for $d(G)$ in [1, 2, 6, 8, 9, 10, 14, 17, 18, 19, 20, 24. To study these notions, various perspectives have been considered in literature, because both measure theory and combinatorial techniques may be applied in order to get restrictions on the structure of a group.

The present paper investigates a similar concept, the permutability degree of $G$

$$
p d(G)=\frac{1}{|G||\mathcal{L}(G)|} \sum_{X \in \mathcal{L}(G)}\left|P_{G}(X)\right|
$$

Date: April 23, 2022.

2010 Mathematics Subject Classification. Primary 20P05; Secondary 06B23, 20D60.

Key words and phrases. Subgroup commutativity degree; commutativity degree; permutability degree; dihedral groups; inequalities.

$\dagger$ Institute of Mathematics and Informatics, Vilnius University, Vilnius, Lithuania; email: daniele.otera@gmail.com

$\dagger \dagger$ Department of Mathematics and Applied Mathematics, University of Cape Town, Cape Town, South Africa; email: francescog.russo@yahoo.com. 
and its connections with $s d(G)$ and $d(G)$. In the previous formula, the permutizer $P_{G}(X)$ of a subgroup $X$ of $G$ is defined to be the subgroup generated by all cyclic subgroups of $G$ that permute with $X$, that is, $P_{G}(X)=\langle g \in G \mid\langle g\rangle X=X\langle g\rangle\rangle$. This means that $X \in \mathcal{L}\left(P_{G}(X)\right)$ and $X \neq P_{G}(X)$ if and only if $X\langle g\rangle=\langle g\rangle X$ for some $g \in G-X$.

We concentrate on permutizers because several classifications are available in literature on this topic. Recall that a group $G$ such that $X \neq P_{G}(X)$ for every proper subgroup $X$ of $G$ is said to satisfy the permutizer condition $\mathbf{P}$, or briefly $\mathbf{P}$-group. Therefore the permutizer condition generalizes the well-known normalizer condition (see [26]) and gives information on how the group is near to be supersolvable. The study of permutizers is not new and it is based on a series of fundamental contributions [3, 21, 23, 30] in the last 20 years. From [3, Corollary $2]$, we know that for groups of odd order the permutizer condition is equivalent of being supersolvable and actually, a complete classification of $\mathbf{P}$-groups can be found in 3 .

Now we may define the subgroup

$$
P(G)=\bigcap_{H \in \mathcal{L}(G)} P_{G}(H)
$$

and correlate it with other subgroups of $G$. For instance, it is easy to check that the norm $N(G)$ of $G$ (see [26] for the properties of $N(G)$ ) satisfies the following relation

$$
Z(G)=\bigcap_{x \in G} C_{G}(x) \subseteq N(G)=\bigcap_{H \in \mathcal{L}(G)} N_{G}(H) \subseteq \bigcap_{H \in \mathcal{L}(G)} P_{G}(H)=P(G) .
$$

This relation emphasizes how $P(G)$ is connected with other subgroups, widely investigated in literature, such as intersections of normalizers or of centralizers. Note that for any $X \in \mathcal{L}(P(G))$ one has $P_{G}(X)=G$.

The subgroup $P(G)$ is also important because it allows us to "manipulate" the expression of $p d(G)$, for getting some analogies with

$$
s d(G)=\frac{1}{|\mathcal{L}(G)|^{2}} \sum_{H \in \mathcal{L}(G)}\left|\mathcal{C}_{\mathcal{L}(G)}(H)\right| \text { and } d(G)=\frac{1}{|G|^{2}} \sum_{x \in G}\left|C_{G}(x)\right|,
$$

where $P_{G}(X)$ is the natural substitute of $\mathcal{C}_{\mathcal{L}(G)}(X)=\{Y \in \mathcal{L}(G) \mid Y X=X Y\}$ in [22, 27] and of $C_{G}(x)=\{y \in G \mid x y=y x\}$ in 2, 8. This manipulation of the expression of $p d(G)$ will allow us to detect whether $P(G)$ is cyclic or not by looking only at the size of $p d(G)$.

\section{BASIC PROPERTIES AND TERMINOLOGY}

Some of the following observations will be useful later on.

Remark 2.1. Since $P_{G}(X)$ is a subgroup of $G$, for all $X \in \mathcal{L}(G)$, and it always contains the trivial subgroup, then $\left|P_{G}(X)\right| \leq|G|$ and also

$$
0<\sum_{X \in \mathcal{L}(G)}\left|P_{G}(X)\right| \leq|\mathcal{L}(G)||G|
$$

so that $p d(G) \in] 0,1]$. 
Remark 2.2. A group $G$ has $p d(G)=1$ if and only if the sum of all $\left|P_{G}(X)\right|$ for $X \in \mathcal{L}(G)$ is equal to $|G||\mathcal{L}(G)|$. By default, a quasihamiltonian group $G$, that is, a group in which every subgroup is permutable, has $p d(G)=1$. A classification of quasihamiltonian groups can be found in [26. Theorems 2.4.11 and 2.4.16] and, roughly speaking, these groups are direct products of abelian groups by a copy of the quaternion group of order 8 . In particular, abelian groups have permutability degree equal to 1.

Another case in which the permutability degree reaches 1 is the following.

Remark 2.3. A $\mathbf{P}$-group $G$ in which all proper subgroups are maximal has $p d(G)=$ 1. In such a case for all proper subgroups $X$ of $G$ one has $X \subset P_{G}(X)=G$ and so $p d(G)=1$. One might be tempted to think that all $\mathbf{P}$-groups have permutability degree equal to 1, but Example 3.2 below shows this is false, and then the additional condition "in which all proper subgroups are maximal" cannot be omitted.

Now we rewrite the original expression of permutability degree in the following more useful form. Since $\mathcal{L}(P(G))$ is a sublattice of $\mathcal{L}(G)$, it turns out that

$$
p d(G)=\frac{1}{|G||\mathcal{L}(G)|}\left(\sum_{X \in \mathcal{L}(P(G))}\left|P_{G}(X)\right|+\sum_{X \in \mathcal{L}(G)-\mathcal{L}(P(G))}\left|P_{G}(X)\right|\right) .
$$

Note also that a cyclic group $C$ (or better a quasihamiltonian group $Q$ ) has $s d(C)=p d(C)=d(C)=1$ (or better $s d(Q)=p d(Q)=1$ ). Therefore, relations between $s d(G)$ and $p d(G)$ are meaningful when $G$ is noncylic and nonquasihamiltonian (see Remark 2.2).

For the sake of completeness, we recall some results of Beidleman and Heineken in 44. The quasicenter $Q(G)$ of $G$ is the subgroup of $G$ generated by all elements $g \in G$ such that $\langle g\rangle K=K\langle g\rangle$, where $K$ is an arbitrary subgroup of $G$. The subgroup $Q(G)$ was introduced by Mukherjee and studied by several authors in the last years (see [4, 5, 25]), who investigated chains of quasicenters and relations with supersolvable groups. On the other hand, the hyperquasicenter of $G$, denoted by $Q_{\infty}(G)$, is the largest term of the chain $1=Q_{0}(G) \leq Q_{1}(G)=Q(G) \leq$ $\ldots \leq Q_{i}(G) \leq Q_{i+1}(G) \leq \ldots$ of normal subgroups of $G$, where, for any $i \geq 0$, $Q_{i+1}(G) / Q_{i}(G)=Q\left(G / Q_{i}(G)\right)$ and $Q_{\infty}(G)=\bigcup_{i \geq 0} Q_{i}(G)$.

Recall that a normal subgroup $N$ of $G$ is said to be hypercyclically embedded in $G$ if it contains a $G$-invariant series whose factors are cyclic. It is easy to see that $G$ contains a unique largest hypercyclically embedded subgroup, which we denote $\Sigma(G)$. More precisely, [4, Theorem 1] shows that $\Sigma(G)=Q_{\infty}(G)$ is true for any group $G$. Some interesting connections hold between $\mathbf{P}$-groups, $P(G)$ and $Q_{\infty}(G)$. For instance, [3, (3.1), p. 697] shows that a group $G$ is a $\mathbf{P}$-group if and only if $G / \Sigma(G)$ is a $\mathbf{P}$-group. As a first consequence, a group $G$ is a $\mathbf{P}$-group if and only if $G / Q_{\infty}(G)$ is a $\mathbf{P}$-group. As a second consequence, $Z(G) \subseteq Q(G) \subseteq P(G)$ is true for any group $G$. Furthermore, $Q_{\infty}(G)=P(G)$ if and only if $P(G)=\Sigma(G)$.

\section{EXAMPLES}

Now we specify some of the previous notions for the symmetric group $S_{3}$ on 3 objects. This will help us to visualize analogies and differences between permutability degrees, subgroup commutativity degrees and commutativity degrees. 
Example 3.1. The smallest nonabelian group $S_{3}$ has $\mathcal{L}\left(S_{3}\right)=\left\{\{1\}, S_{3}, A_{3}, H, K, L\right\}$, where $A_{3}=\langle(123)\rangle=\langle a\rangle, H=\langle(12)\rangle=\langle h\rangle, K=\langle(13)\rangle=\langle k\rangle, L=\langle(23)\rangle=\langle l\rangle$. Noting that $H K \neq K H, H L \neq L H, K L \neq L K$, one has

$$
\begin{gathered}
P_{S_{3}}(\{1\})=P_{S_{3}}\left(A_{3}\right)=P_{S_{3}}\left(S_{3}\right)=S_{3} ; \\
P_{S_{3}}(H)=P_{S_{3}}(K)=P_{S_{3}}(L)=S_{3} ; P\left(S_{3}\right)=S_{3} .
\end{gathered}
$$

The fact that $P_{S_{3}}(\{1\})=P_{S_{3}}\left(A_{3}\right)=P_{S_{3}}\left(S_{3}\right)=S_{3}$ is clear, since we are dealing with permutizers of normal subgroups. On the other hand, $A_{3}=\langle a\rangle \subseteq P_{S_{3}}(H)$ by definition. Now, $H \subseteq P_{S_{3}}(H)$ is obvious. Then $H A_{3}$, which is a subgroup of $S_{3}$, should be contained in $P_{S_{3}}(H)$ and $\left|H A_{3}\right|=\frac{|H|\left|A_{3}\right|}{\left|H \cap A_{3}\right|}=6$. This forces $P_{S_{3}}(H)$ to be equal to $S_{3}$. Similarly we get $P_{S_{3}}(K)=P_{S_{3}}(L)=S_{3}$.

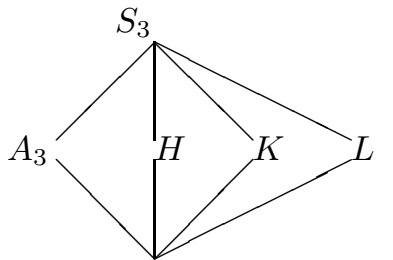

Fig.III.1. Hasse diagram of $\mathcal{L}\left(S_{3}\right)$.

$\{1\}$

It is interesting to note that an example as easy as this has a lot of properties in our perspective of study. The group $S_{3}$ is supersovable by looking at the series $\{1\} \triangleleft A_{3} \triangleleft S_{3}$, but is not quasihamiltonian, due to $H K \neq K H$. At the same time, $S_{3}$ does not satisfy the normalizer condition, since it is not nilpotent. Moreover, $S_{3}$ has $Z\left(S_{3}\right)=\{1\}, \Sigma\left(S_{3}\right)=Q_{2}\left(S_{3}\right)=Q_{\infty}\left(S_{3}\right)=S_{3}, Q\left(S_{3}\right)=A_{3}$ and it is a $\mathbf{P}$-group, since $S_{3} / \Sigma\left(S_{3}\right)=\{1\}$ is obviously a $\mathbf{P}$-group.

A direct calculation shows that

$$
\begin{aligned}
& 6 \cdot 6 \operatorname{pd}\left(S_{3}\right)=\sum_{X \in \mathcal{L}\left(S_{3}\right)}\left|P_{S_{3}}(X)\right|=\left|P_{S_{3}}(H)\right|+\left|P_{S_{3}}(K)\right| \\
& +\left|P_{S_{3}}(L)\right|+\left|P_{S_{3}}\left(A_{3}\right)\right|+\left|P_{S_{3}}\left(S_{3}\right)\right|+\left|P_{S_{3}}(\{1\})\right|=36,
\end{aligned}
$$

then $p d\left(S_{3}\right)=1>s d\left(S_{3}\right)=\frac{5}{6}>\frac{1}{2}=d\left(S_{3}\right)$, agreeing with the computations in [27, p.2510] and [8, 9].

Another easy (but interesting) example is the following.

Example 3.2. The dihedral group of order 8 is $D_{8}=\left\langle a, b \mid a^{2}=b^{4}=1, a^{-1} b a=b^{-1}\right\rangle$ and has

$$
\mathcal{L}\left(D_{8}\right)=\left\{\{1\},\langle b\rangle,\left\langle b^{2}\right\rangle,\langle a\rangle,\langle b a\rangle,\left\langle b^{2} a\right\rangle,\left\langle b^{3} a\right\rangle,\left\{1, b^{2}, a, b^{2} a\right\},\left\{1, b^{2}, b a, b^{3} a\right\}, D_{8}\right\} .
$$

The normal subgroups are $D_{8},\{1\}, B=\langle b\rangle, Z\left(D_{8}\right)=\left\langle b^{2}\right\rangle, M_{1}=\left\{1, b^{2}, a, b^{2} a\right\}$ and $M_{2}=\left\{1, b^{2}, b a, b^{3} a\right\}$. Notice that $H=\left\langle b^{2} a\right\rangle$ and $K=\langle a\rangle$ are contained in $M_{1}$, while $U=\langle b a\rangle$ and $V=\left\langle b^{3} a\right\rangle$ in $M_{2}$. 


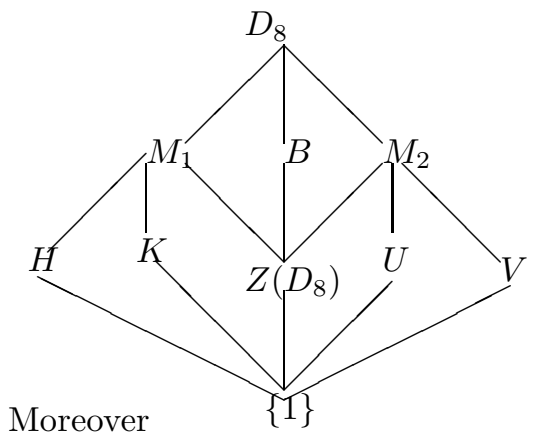

Fig.III.2. Hasse diagram of $\mathcal{L}\left(D_{8}\right)$.

$$
\begin{gathered}
8=\left|D_{8}\right|=\left|P_{D_{8}}(\{1\})\right|=\left|P_{D_{8}}\left(D_{8}\right)\right|=\left|P_{D_{8}}(\langle b\rangle)\right|=\left|P_{D_{8}}\left(Z\left(D_{8}\right)\right)\right| \\
=\left|P_{D_{8}}\left(M_{1}\right)\right|=\left|P_{D_{8}}\left(M_{2}\right)\right|, \\
4=\left|M_{1}\right|=\left|P_{D_{8}}(H)\right|=\left|P_{D_{8}}(K)\right|, \quad 4=\left|M_{2}\right|=\left|P_{D_{8}}(V)\right|=\left|P_{D_{8}}(U)\right|
\end{gathered}
$$

and $Q\left(D_{8}\right)=P\left(D_{8}\right)=Z\left(D_{8}\right)<\Sigma\left(D_{8}\right)=Q_{\infty}\left(D_{8}\right)=D_{8}$. In fact, $D_{8}=\Sigma\left(D_{8}\right)$ is supersolvable and it is also a $\mathbf{P}$-group, but nevertheless its permutability degree is different from 1 , because

$8 \cdot 10 p d\left(D_{8}\right)=\sum_{X \in \mathcal{L}\left(D_{8}\right)}\left|P_{D_{8}}(X)\right|=6 \cdot\left|D_{8}\right|+2 \cdot\left|\left\{1, b^{2}, a, b^{2} a\right\}\right|+2 \cdot\left|\left\{1, b^{2}, b a, b^{3} a\right\}\right|=64$.

More precisely,

$$
d\left(D_{8}\right)=\frac{5}{8}<p d\left(D_{8}\right)=\frac{64}{80}=\frac{4}{5}<s d\left(D_{8}\right)=\frac{46}{55} .
$$

The value of $d\left(D_{8}\right)$ can be found in $\left[8\right.$ and that of $s d\left(D_{8}\right)$ in [27]. This example shows that there exist $\mathbf{P}$-groups with permutability degree different from 1 . Note that $D_{8}$ satisfies $8=\left|D_{8}\right|<\left|\mathcal{L}\left(D_{8}\right)\right|=10$ but

$$
\begin{gathered}
8=\left|\left\{\{1\}, D_{8},\langle b\rangle,\left\{1, b^{2}, b a, b^{3} a\right\},\left\{1, b^{2}, a, b^{2} a\right\},\left\langle b^{2} a\right\rangle,\left\langle b^{2}\right\rangle,\langle a\rangle\right\}\right|=\left|\mathcal{C}_{\mathcal{L}\left(D_{8}\right)}(\langle a\rangle)\right| \\
\mathbb{E}\left|\mathcal{Z}_{\mathcal{L}\left(D_{8}\right)}(\langle a\rangle)\right|=\left|\left\{\{1\},\left\langle b^{2} a\right\rangle,\left\langle b^{2}\right\rangle,\langle a\rangle\right\}\right|=4 .
\end{gathered}
$$

Examples 3.1 and 3.2 illustrate a series of problems for the computation of the permutability degree, arising from the nature of the subgroup lattice of the groups under consideration. We will come back to this point later on.

\section{General properties of the permutability degree}

We note that [8, Theorems $2.5,3.3$ ] shows that the commutativity degree is monotone. This is a well-known property, which is due to the fact that we are dealing with a positive monotone measure of probability. Similar situations can be found for $\operatorname{sd}(G)$ in [27, Proposition 2.4, Corollaries 2.5, 2.6, 2.7, Theorems 3.1.1, $3.1 .5]$ and in [9, 14, 22. For $p d(G)$ we have something similar.

Theorem 4.1. Let $H$ be a subgroup of a group $G$. Then

$$
\frac{|\mathcal{L}(H)|}{|\mathcal{L}(G)||G: H|} p d(H) \leq p d(G)
$$

Moreover, if $\left|P_{G}(X): P_{H}(X)\right| \leq|G: H|$ for all $X \in \mathcal{L}(G), P(G) \leq H$ and $|\mathcal{L}(G)-\mathcal{L}(P(G))| \leq|\mathcal{L}(P(G))|$, then $|\mathcal{L}(G)| p d(G) \leq 2|\mathcal{L}(H)| p d(H)$. In particular,

$$
\frac{|\mathcal{L}(H)|}{|\mathcal{L}(G)||G: H|} p d(H) \leq p d(G) \leq \frac{2|\mathcal{L}(H)|}{|\mathcal{L}(G)|} p d(H)
$$


Proof. We start by proving the first inequality. Since $\left|P_{H}(X)\right| \leq\left|P_{G}(X)\right|$ for all $X \in \mathcal{L}(G)$, we have

$$
\begin{gathered}
|G||\mathcal{L}(G)| p d(G)=\sum_{X \in \mathcal{L}(G)}\left|P_{G}(X)\right| \\
\geq \sum_{X \in \mathcal{L}(G)}\left|P_{H}(X)\right|=\sum_{X \in \mathcal{L}(H)}\left|P_{H}(X)\right|=p d(H)|H||\mathcal{L}(H)|
\end{gathered}
$$

and the result follows.

Now we prove the second inequality. Since by hypothesis, $\left|P_{G}(X): P_{H}(X)\right| \leq$ $|G: H|$, we have $\left|P_{G}(X)\right| \leq|G: H|\left|P_{H}(X)\right|$ and so

$$
|G||\mathcal{L}(G)| p d(G)=\sum_{X \in \mathcal{L}(G)}\left|P_{G}(X)\right| \leq|G: H| \sum_{X \in \mathcal{L}(G)}\left|P_{H}(X)\right|
$$

but, from (2.1), this means

$$
=|G: H|\left(\sum_{X \in \mathcal{L}(P(G))}\left|P_{H}(X)\right|+\sum_{X \in \mathcal{L}(G)-\mathcal{L}(P(G))}\left|P_{H}(X)\right|\right)
$$

and the inequality $|\mathcal{L}(G)-\mathcal{L}(P(G))| \leq|\mathcal{L}(P(G))|$ provided by hypothesis, implies

$$
\begin{gathered}
\leq|G: H|\left(\sum_{X \in \mathcal{L}(P(G))}\left|P_{H}(X)\right|+\sum_{X \in \mathcal{L}(P(G))}\left|P_{H}(X)\right|\right)=2|G: H| \sum_{X \in \mathcal{L}(P(G))}\left|P_{H}(X)\right| \\
\leq 2|G: H| \sum_{X \in \mathcal{L}(H)}\left|P_{H}(X)\right|=2|G: H||H||\mathcal{L}(H)| p d(H)
\end{gathered}
$$

from which we have $|\mathcal{L}(G)| p d(G) \leq 2|\mathcal{L}(H)| p d(H)$.

A classic splitting result for the product probability of two independent events is described by the following corollary. The proof may be generalized to finitely many factors, whose orders are pairwise coprime.

Proposition 4.2. Let $G$ and $H$ be two groups such that $\operatorname{gcd}(|G|,|H|)=1$. Then $p d(G \times H)=p d(G) p d(H)$.

Proof. Given three groups $A, B$ and $C$ such that $A \times B \subseteq C$, we know that $N_{C}(A \times B)=N_{C}(A) \times N_{C}(B)$. This holds similarly for the permutizers and it is easy to see that $P_{C}(A \times B)=P_{C}(A) \times P_{C}(B)$. Now this fact and the assumption $\operatorname{gcd}(|G|,|H|)=1$ allow us to conclude that

$$
\begin{aligned}
& \frac{1}{|G \times H||\mathcal{L}(G \times H)|} \sum_{X \times Y \in \mathcal{L}(G \times H)}\left|P_{G \times H}(X \times Y)\right| \\
= & \frac{1}{|G||\mathcal{L}(G)|} \frac{1}{|H||\mathcal{L}(H)|} \sum_{X \in \mathcal{L}(G)}\left|P_{G}(X)\right| \sum_{Y \in \mathcal{L}(H)}\left|P_{H}(Y)\right| .
\end{aligned}
$$

The underlying problem we deal with is the order of the subgroup lattices, which is hard to predict in general. If we concentrate on some groups arising from finite geometries, then the situation is more clear (dihedral groups, semidihedral groups and generalized quaternion groups were studied in 2 , $2,11,22,27,28,29$ from a 
similar perspective). Having in mind Examples 3.1 and 3.2, we observe from 26, pp. 26-29] and [22, 27] that the dihedral group

$$
D_{2 n}=\left\langle x, y \mid x^{2}=y^{n}=1, x^{-1} y x=y^{-1}\right\rangle=C_{2} \ltimes C_{n}=\langle x\rangle \ltimes\langle y\rangle
$$

of symmetries of a regular polygon with $n \geq 1$ edges has order $2 n$ and splits in the semidirect product of a cyclic group $\langle y\rangle \simeq C_{n}$ of order $n$ by a cyclic group $\langle x\rangle \simeq C_{2}$ of order 2 acting by inversion on $C_{n}$. In particular, $S_{3} \simeq D_{6}$ for $n=3$ and one can note that the Hasse diagram of $\mathcal{L}\left(D_{6}\right)=\mathcal{L}\left(S_{3}\right)$ forms a diamond in which there are only 4 atomic elements (see [26] for this terminology) in between $\{1\}$ and $D_{6}$ and their number can be easily computed. The following Fig.III.3 summarizes the information of Example 3.1 in a more general situation.

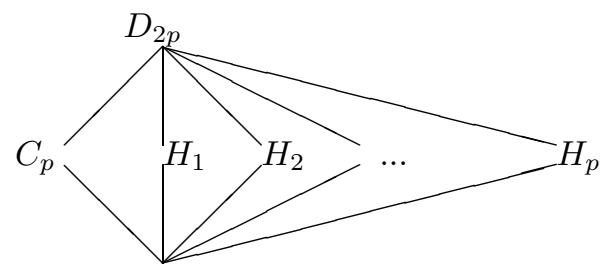

$\{1\}$

Fig.III.3. Hasse diagram of $\mathcal{L}\left(D_{2 p}\right)$ with odd prime $p \geq 3$;

$$
H_{1} \simeq H_{2} \simeq \ldots \simeq H_{p} \simeq C_{2} .
$$

From Figs.III.1, III.2 and III.3, it is clear that $\mathcal{L}\left(D_{2 p}\right)$ has $p+1$ proper subgroups, and this fact comes out from the following formula

$$
\left|\mathcal{L}\left(D_{2 n}\right)\right|=\sigma(n)+\tau(n),
$$

where $\sigma(n)$ and $\tau(n)$ are the sum and the number of all divisors of $n$ (here $n$ is arbitrary, not necessarily an odd prime), respectively.

In particular, if $n=p^{m}$ is a power of a prime $p$ (possibly $p=2$ ) for some $m \geq 0$, then the set of all divisors of $p^{m}$ is $\operatorname{Div}\left(p^{m}\right)=\left\{1, p, p^{2}, \ldots, p^{m}\right\}$ so that

$$
\sigma\left(p^{m}\right)=\sum_{j=0}^{m} p^{j}=\frac{1-p^{m+1}}{1-p} \text { and } \tau\left(p^{m}\right)=\left|\operatorname{Div}\left(p^{m}\right)\right|=m+1 .
$$

The reader has probably noted that we used the formula for the sum of a geometric series in the previous expression for $\sigma\left(p^{m}\right)$. Then we may conclude that

$$
\left|\mathcal{L}\left(D_{2 p^{m}}\right)\right|=1+m+\frac{1-p^{m+1}}{1-p}=m+\frac{p^{m+1}+p-2}{p-1} .
$$

The next result shows an upper bound for $p d(G)$, when $|\mathcal{L}(G)|$ is of type (4.4).

Theorem 4.3. Let $G$ be a noncyclic group and $p$ the smallest prime divisor of $|G|$. If $|P(G)|=p$ and $|\mathcal{L}(G)|=m+\frac{p^{m+1}+p-2}{p-1}$ for some $m \geq 0$, then

$$
p d(G) \leq \frac{p^{m+1}+2 p^{2}+(m-3) p-m}{p^{m+2}+(m+1) p^{2}-(m+2) p} .
$$

Proof. If $P_{G}(X)=G$ for some $X \in \mathcal{L}(G)$, then $|G|=\left|P_{G}(X)\right|=|P(G)|$ and $G$ would be cyclic, contradicting our assumption. Without loss of generality we 
may assume $P_{G}(X) \neq G$ for all $X \in \mathcal{L}(G)$. The minimality of $p$ implies that $\left|P_{G}(X)\right| \leq \frac{|G|}{p}$ for all $X \in \mathcal{L}(G)$. Of course, $|\mathcal{L}(P(G))|=2$ and (2.1) becomes

$$
\begin{gathered}
|G| \cdot\left(\frac{m p-m+p^{m+1}+p-2}{p-1}\right) \cdot p d(G)=2|G|+\sum_{X \in \mathcal{L}(G)-\mathcal{L}(P(G))}\left|P_{G}(X)\right| \\
\leq 2|G|+(|\mathcal{L}(G)-\mathcal{L}(P(G))|) \frac{|G|}{p} \\
=2|G|+\frac{|G|}{p}(|\mathcal{L}(G)|-2)=2|G|+\frac{|G|}{p}\left(\frac{m p-m+p^{m+1}+p-2}{p-1}-2\right) \\
=\frac{|G|}{p}\left(\frac{m p-m+p^{m+1}+p-2-2 p+2+2 p^{2}-2 p}{p-1}\right) \\
=\frac{|G|}{p}\left(\frac{p^{m+1}+2 p^{2}+(m-3) p-m}{p-1}\right) .
\end{gathered}
$$

This gives, as claimed.

\section{Some theorems of Structure}

The present section is devoted to prove restrictions on $P(G)$, arising from exact bounds for $p d(G)$, when $G$ is an arbitrary group. The evidences of Examples 3.1 and 3.2 motivated most of the following results.

Theorem 5.1. Let $G$ be a group (with $p d(G) \neq 1$ ) and $p$ the smallest prime divisor of $|G|$. Then

$$
\left(1-\frac{p}{|G|}\right) \frac{|\mathcal{L}(P(G))|}{|\mathcal{L}(G)|}+\frac{p}{|G|} \leq p d(G) .
$$

Moreover, if $P_{G}(X)$ is a proper subgroup of $G$ for all $X \in \mathcal{L}(G)-\mathcal{L}(P(G))$, then

$$
p d(G) \leq \frac{1}{p}+\frac{(p-1)|\mathcal{L}(P(G))|}{p|\mathcal{L}(G)|} .
$$

Proof. In order to prove the lower bound, it is enough to note from (2.1) that

$$
\begin{gathered}
|\mathcal{L}(G)||G| p d(G)=|\mathcal{L}(P(G))||G|+\sum_{X \in \mathcal{L}(G)-\mathcal{L}(P(G))}\left|P_{G}(X)\right| \\
\geq|\mathcal{L}(P(G))||G|+(|\mathcal{L}(G)|-|\mathcal{L}(P(G))|) p,
\end{gathered}
$$

where we have used in the last step that $\left|P_{G}(X)\right| \geq p$. Then we continue

$$
=(|G|-p)|\mathcal{L}(P(G))|+p|\mathcal{L}(G)|
$$

from which we get

$$
p d(G) \geq \frac{(|G|-p)|\mathcal{L}(P(G))|+p|\mathcal{L}(G)|}{|\mathcal{L}(G)||G|}=\frac{(|G|-p)|\mathcal{L}(P(G))|}{|\mathcal{L}(G)||G|}+\frac{p}{|G|} .
$$

Now we prove the upper bound. Formula (2.1) becomes again (5.1)

$$
|G||\mathcal{L}(G)| p d(G)=|\mathcal{L}(P(G))||G|+\sum_{X \in \mathcal{L}(G)-\mathcal{L}(P(G))}\left|P_{G}(X)\right|
$$


once one uses the fact that $P_{G}(X)=G$ for every $X \in \mathcal{L}(P(G))$. Now, since $\left|G: P_{G}(X)\right| \neq 1$ for every $X \in \mathcal{L}(G)-\mathcal{L}(P(G))$, we get $\left|G: P_{G}(X)\right| \geq p$, that is, $\left|P_{G}(X)\right| \leq \frac{|G|}{p}$. Therefore (5.1) is upper bounded by

$$
\leq|\mathcal{L}(P(G))||G|+(|\mathcal{L}(G)|-|\mathcal{L}(P(G))|) \frac{|G|}{p}=\frac{(p-1)|\mathcal{L}(P(G))||G|}{p}+\frac{|\mathcal{L}(G)||G|}{p}
$$

and the result follows.

Of course, $D_{8}$ satisfies the lower bound, but not the upper bound, of Theorem 5.1. Details can be deduced from the information of Example 3.2 This is to justify that Theorem 5.1 originates from evidences of computational nature. On the other hand, Example 3.2 shows also that $Z\left(D_{8}\right)=P\left(D_{8}\right) \simeq C_{2}$. Then, when can we say that $P\left(D_{8}\right)$ is noncyclic? The next two results concern this question.

Theorem 5.2. If $P(G)$ is a nontrivial proper subgroup of a group $G$ and $p d(G)=$ $\frac{1}{2}+\frac{|\mathcal{L}(P(G))|}{2|\mathcal{L}(G)|}$, then $P(G)$ is noncyclic.

Proof. By assumption we exclude the cases $P(G)=G$ and $P(G)=\{1\}$, which are the extremal situations already known. Assume that $P(G)$ is cyclic of prime order $q \geq p \geq 2$, where $p$ is the smallest prime dividing $|G|$. We may apply the arguments of the proof of Theorem 4.3 and, noting that $|\mathcal{L}(P(G))|=2$, we find that

$$
\begin{aligned}
p d(G)=\frac{1}{2}+\frac{|\mathcal{L}(P(G))|}{2|\mathcal{L}(G)|}=\frac{1}{2}+ & \frac{1}{|\mathcal{L}(G)|}=\frac{|\mathcal{L}(G)|+2}{2|\mathcal{L}(G)|} \leq \frac{2|G|}{|G||\mathcal{L}(G)|}+\frac{|G|(|\mathcal{L}(G)|-2)}{p|G||\mathcal{L}(G)|} \\
& =\frac{(2 p+|\mathcal{L}(G)|-2)|G|}{p|G||\mathcal{L}(G)|}
\end{aligned}
$$

and then the inequality

$$
\frac{|\mathcal{L}(G)|+2}{2}=\frac{|\mathcal{L}(G)|}{2}+1 \leq \frac{(2 p-2)}{p}+\frac{|\mathcal{L}(G)|}{p}
$$

which means

$$
\frac{|\mathcal{L}(G)|}{2}-\frac{\mid \mathcal{L}(G)}{p}=\frac{(p-2)|\mathcal{L}(G)|}{2 p} \leq 1-\frac{2}{p}=\frac{p-2}{p} .
$$

From this we derive the contradiction $\frac{|\mathcal{L}(G)|}{2} \leq 1$, as at least $\{1\}$ and $G$ are contained in $\mathcal{L}(G)$. Therefore, $P(G)$ cannot be cyclic of prime order and we may assume that $P(G)$ is cyclic of order $k \geq 2$. Now, we note that $|\mathcal{L}(P(G))|=|\operatorname{Div}(k)|$, where $\operatorname{Div}(k)$ is the set of all divisors of $k$. Here the argument we just used for $q$ may still be applied. In fact we have

$$
\begin{gathered}
\frac{1}{2}+\frac{|\operatorname{Div}(k)|}{2|\mathcal{L}(G)|}=\frac{|\mathcal{L}(G)|+|\operatorname{Div}(k)|}{2|\mathcal{L}(G)|} \leq \frac{|\operatorname{Div}(k)||G|}{|G||\mathcal{L}(G)|}+\frac{|G|(|\mathcal{L}(G)|-|\operatorname{Div}(k)|)}{p|G||\mathcal{L}(G)|} \\
=\frac{(|\operatorname{Div}(k)| p+|\mathcal{L}(G)|-|\operatorname{Div}(k)|)|G|}{p|G| \mathcal{L}(G) \mid}
\end{gathered}
$$

then

$$
\frac{|\mathcal{L}(G)|+|\operatorname{Div}(k)|}{2}=\frac{|\mathcal{L}(G)|}{2}+\frac{|\operatorname{Div}(k)|}{2} \leq \frac{(p-1)|\operatorname{Div}(k)|}{p}+\frac{|\mathcal{L}(G)|}{p}
$$

which means

$$
\frac{|\mathcal{L}(G)|}{2}-\frac{\mid \mathcal{L}(G)}{p}=\frac{(p-2)|\mathcal{L}(G)|}{2 p} \leq \frac{(p-1)|\operatorname{Div}(k)|}{p}
$$


and this would imply that $|\mathcal{L}(G)| \leq|\operatorname{Div}(k)|=|\mathcal{L}(P(G))|$, that is, $\mathcal{L}(G) \subseteq \mathcal{L}(P(G))$ and then $\mathcal{L}(G)=\mathcal{L}(P(G))$. This condition implies $G=P(G)$, a contradiction.

The reader may note that Theorem 5.2 describes a very general situation, which cannot be reduced to those in Examples 3.1 and 3.2. In fact, looking at Example 3.1. $P\left(D_{6}\right)=D_{6}$ and so $P\left(D_{6}\right)$ is not a proper subgroup of $D_{6}$, and this means that one of the assumptions of Theorem 5.2 is not satisfied. On the other hand, $P\left(D_{8}\right)=Z\left(D_{8}\right)$ is a nontrivial proper subgroup of $D_{8}$ (see Example 3.2), but $\frac{4}{5}=p d\left(D_{8}\right) \neq \frac{1}{2}+\frac{1}{10}=\frac{3}{5}$, and in fact $P\left(D_{8}\right)$ is cyclic. Once again, Theorem 5.2 can not be applied. These two examples show that we cannot strengthen further Theorem 5.2 ,

However, we may detect groups $G$ with cyclic $P(G)$. The following result shows this circumstance.

Theorem 5.3. Let $P(G)$ be a nontrivial proper subgroup of a group $G$ with $p d(G)=$ $\frac{4}{5}$ and $p$ be the smallest prime divisor of $|G|$. If $\frac{4|G|-5 p}{5|G|-5 p} \leq \frac{2}{|\mathcal{L}(G)|}$, then $P(G)$ is cyclic of prime order.

Proof. From the lower bound of Theorem [5.1, we have

$$
\begin{aligned}
p d(G)=\frac{4}{5} & \geq\left(1-\frac{p}{|G|}\right) \frac{|\mathcal{L}(P(G))|}{|\mathcal{L}(G)|}+\frac{p}{|G|} \Leftrightarrow \frac{\frac{4}{5}}{1-\frac{p}{|G|}}-\frac{\frac{p}{|G|}}{1-\frac{p}{|G|}} \geq \frac{|\mathcal{L}(P(G))|}{|\mathcal{L}(G)|} \\
& \Leftrightarrow \frac{\frac{4|G|-5 p}{5|G|}}{\frac{|G|-p}{|G|}} \geq \frac{|\mathcal{L}(P(G))|}{|\mathcal{L}(G)|} \Leftrightarrow \frac{4|G|-5 p}{5|G|-5 p} \geq \frac{|\mathcal{L}(P(G))|}{|\mathcal{L}(G)|} .
\end{aligned}
$$

We conclude that $\frac{|\mathcal{L}(P(G))|}{|\mathcal{L}(G)|} \leq \frac{2}{|\mathcal{L}(G)|}$, hence $|\mathcal{L}(P(G))| \leq 2$. This forces $P(G)$ to be cyclic of prime order.

\section{Computations for dihedral groups}

We describe an instructive example, which correlates most of the notions which we have seen until now.

Proposition 6.1. Let $p$ be an odd prime. Then

$$
1=p d\left(D_{2 p}\right)>s d\left(D_{2 p}\right)=\frac{7 p^{3}-5 p^{2}-11 p+9}{p^{4}+4 p^{3}-2 p^{2}-12 p+9}>\frac{p+3}{4 p}=d\left(D_{2 p}\right) .
$$

Proof. Noting that $D_{2 p}=C_{2} \ltimes C_{p}$ (see (4.1)) and that $\mathcal{L}\left(D_{2 p}\right)$ forms a diamond (as in Fig.III.3), we conclude that $Z\left(D_{2 p}\right)$ is trivial and $C_{D_{2 p}}\left(C_{p}\right)=C_{p}$ is the unique maximal normal subgroup of $D_{2 p}$. Moreover $D_{2 p}$ is a $\mathbf{P}$-group, because $Q_{\infty}\left(D_{2 p}\right)=D_{2 p}$. Therefore a proper subgroup $H$ of $D_{2 p}$ should be properly contained in $P_{D_{2 p}}(H)$ and necessarily $P_{D_{2 p}}(H)=D_{2 p}$. Thus, we find that $p d\left(D_{2 p}\right)=1$.

On the other hand, we may specialize the formula

$$
s d\left(D_{2 p}\right)=\frac{\tau(p)^{2}+2 \tau(p) \sigma(p)+g(p)}{(\tau(p)+\sigma(p))^{2}},
$$

given in [27, Theorem 3.1.1], where

$$
g(p)=\frac{3 p^{3}-5 p^{2}+p+1}{p^{2}-2 p+1}
$$


is the arithmetic function in [27, Eq. 10, p.2514]. From (4.3) we deduce $\tau(p)=2$ and $\sigma(p)=p+1$ so that

$$
\begin{gathered}
s d\left(D_{2 p}\right)=\frac{4+2 \cdot 2 \cdot(p+1)+\frac{3 p^{3}-5 p^{2}+p+1}{p^{2}-2 p+1}}{p^{2}+6 p+9}=\frac{8+4 p+\frac{3 p^{3}-5 p^{2}+p+1}{p^{2}-2 p+1}}{p^{2}+6 p+9} \\
=\frac{\frac{8 p^{2}-16 p+8+4 p^{3}-8 p^{2}+4 p+3 p^{3}-5 p^{2}+p+1}{p^{2}-2 p+1}}{p^{2}+6 p+9} \\
=\frac{7 p^{3}-5 p^{2}-11 p+9}{\left(p^{2}+6 p+9\right)\left(p^{2}-2 p+1\right)}=\frac{7 p^{3}-5 p^{2}-11 p+9}{p^{4}+4 p^{3}-2 p^{2}-12 p+9} .
\end{gathered}
$$

Now [20, Remark 4.2] shows that $d\left(D_{2 p}\right)=\frac{p+3}{4 p}$ and for all odd primes we have

$$
\begin{gathered}
0>p^{5}-21 p^{4}+30 p^{3}+26 p^{2}-63 p+27 \\
\Leftrightarrow 28 p^{4}-20 p^{3}-44 p^{2}+36 p>p^{5}+7 p^{4}+10 p^{3}-18 p^{2}-27 p+27 \\
\Leftrightarrow 28 p^{4}-20 p^{3}-44 p^{2}+36 p>p^{5}+4 p^{4}-2 p^{3}-12 p^{2}+9 p+3 p^{4}+12 p^{3}-6 p^{2}-36 p+27 \\
\Leftrightarrow 4 p\left(7 p^{3}-5 p^{2}-11 p+9\right)>(p+3)\left(p^{4}+4 p^{3}-2 p^{2}-12 p+9\right) \\
\Leftrightarrow \frac{7 p^{3}-5 p^{2}-11 p+9}{p^{4}+4 p^{3}-2 p^{2}-12 p+9}>\frac{p+3}{4 p} .
\end{gathered}
$$

The result follows.

From Proposition 6.1, $p d\left(D_{2 p}\right)$ has a constant value for all odd primes, while $s d\left(D_{2 p}\right)$ and $d\left(D_{2 p}\right)$ are functions of $p$. This is an important difference of the permutability degree with respect to the subgroup commutativity degree and the commutativity degree. This reflects the fact that we are looking at permutizers in a group, and not at centralizers.

\section{Some OPEN QUESTIONS}

We end with a series of open questions. They arise naturally from the study of the present subject. The first is motivated by the families of dihedral groups, analyzed in Section 6 (and in other parts of the present paper). Most of these groups may be described in terms of product of groups.

Open Question 7.1. Let $G=N H$ be a product of a normal subgroup $N$ by a subgroup $H$. What can be said about the permutability degree of $G$ ?

It is well known that most of dihedral groups, generalized quaternion groups and semidihedral groups has this structure. They present further analogies in terms of central quotients: one, for instance, is that $D_{8} / Z\left(D_{8}\right) \simeq Q_{8}$ and, roughly speaking, one can generalize this isomorphism to $Q_{2^{4}}=Q_{16}, Q_{2^{5}}=Q_{32}$ and so on. The reader may refer to [15, 16, for recent studies on these groups. Therefore:

Open Question 7.2. What is the permutability degree of generalized quaternion groups and semidihedral groups?

There is also another question, which is more general and may require some computational efforts. A classical result of Cayley allows us to embedd a group in a suitable symmetric group. The knowledge of symmetric groups plays in fact a fundamental role in several aspects of the theory of groups. Therefore:

Open Question 7.3. What is the permutability degree of the symmetric group $S_{n}$ on $n$ objects? 
Finally, the classification of (finite) simple groups may provide interesting aspects of study. For the (finite) simple (and almost simple) groups of sporadic type a lot is known about their subgroup lattices, see [7. Therefore

Open Question 7.4. What is the permutability degree of (finite) simple groups?

\section{ACKNOWLEDGEMENTS}

The first author was partially supported by GNSAGA of INdAM (Italy). The research of the second author is supported in part by NRF (South Africa) for the Grant No. 93652 and in part from the Launching Grant No. 459235 of the University of Cape Town (South Africa). He also thanks Universidade Federal do Rio de Janeiro (Rio de Janeiro, Brazil), where the present subject was originally investigated via the project CAPES No. 061/2013. Finally, we thank the editor for his valuable work and the referee for the questions in Section 7 .

\section{REFERENCES}

[1] A.M. Alghamdi, D.E. Otera and F.G. Russo, On some recent investigations of probability in group theory, Boll. Mat. Pura Appl. 3 (2010), 87-96.

[2] A.M. Alghamdi and F.G. Russo, A generalization of the probability that the commutator of two group elements is equal to a given element, Bull. Iran. Math. Soc. 38 (2012), 973-986.

[3] J.C. Beidleman and D.J. Robinson, On finite groups satisfying the permutizer condition, J. Algebra 191 (1997), 686-703.

[4] J.C. Beidleman and H. Heineken, On the hyperquasicenter of a group, J. Group Theory 4 (2001), 199-206.

[5] J.C. Beidleman, H. Heineken and F.G. Russo, Generalized hypercenters in infinite groups, Asian-Eur. J. Math. 4 (2011), 21-30.

[6] S. Blackburn, J.R. Britnell and M. Wildon, The probability that a pair of elements of a finite group are conjugate, J. London Math. Soc. 86 (2012), 755-778.

[7] T. Connor and D. Leemans, An atlas of subgroup lattices of finite almost simple groups, Ars Math. Contemp. 8 (2015), 259-266.

[8] A. Erfanian, P. Lescot and R. Rezaei, On the relative commutativity degree of a subgroup of a finite group, Comm. Algebra 35 (2007), 4183-4197.

[9] A. Erfanian, R. Rezaei and F.G. Russo, Relative $n$-isoclinism classes and relative nilpotency degree of finite groups, Filomat 27 (2013), 365-369.

[10] A. Erfanian and F.G. Russo, Probability of the mutually commuting $n$-tuples in some classes of compact groups, Bull. Iranian Math. Soc. 34 (2008), 27-37.

[11] M. Farrokhi and F. Saeedi, Factorization numbers of some finite groups, Glasg. Math. J. 54 (2012), 345-354.

[12] M. Farrokhi, Factorization numbers of finite abelian groups, Int. J. Group Theory 2 (2013), $1-8$.

[13] M. Farrokhi and F. Saeedi, Subgroup permutability degree of $\operatorname{PSL}\left(2, p^{n}\right)$, Glasg. Math. J. 55 (2013), 581-590.

[14] R. M. Guralnick and G. R. Robinson, On the commuting probability in finite groups, J. Algebra 300 (2006), 509-528.

[15] H. Heineken and F.G. Russo, On a notion of breadth in the sense of Frobenius, J. Algebra 424 (2015), 208-221.

[16] H. Heineken and F.G. Russo, Groups described by element numbers, Forum Math., to appear, DOI: $10.1515 /$ forum-2013-6005.

[17] K.H. Hofmann and F.G. Russo, The probability that $x$ and $y$ commute in a compact group, Math. Proc. Cambridge Phil. Soc. 153 (2012), 557-571.

[18] K.H. Hofmann and F.G. Russo, The probability that $x^{m}$ and $y^{n}$ commute in a compact group, Bull. Aust. Math. Soc. 87 (2013), 503-513.

[19] P. Lescot, Isoclinism classes and commutativity degrees of finite groups, J. Algebra 177 (1995), 847-869. 
[20] P. Lescot, Central extensions and commutativity degree, Comm. Algebra 29 (2001), 44514460.

[21] X. Liu and Y. Wang, Implications of permutizers of some subgroups in finite groups, Comm. Algebra 33 (2005), 559-565.

[22] D.E. Otera and F.G. Russo, Subgroup S-commutativity degrees of finite groups, Bull. Belgian Math. Soc. 19 (2012), 373-382.

[23] S. Qiao, G. Qian and Y. Wang, Influence of permutizers of subgroups on the structure of finite groups, J. Pure Appl. Algebra 212 (2008), 2307-2313.

[24] F.G. Russo, A probabilistic meaning of certain quasinormal subgroups, Int. J. Algebra 1 (2007), 385-392.

[25] F.G. Russo, On a result of Mukherjee, Int. Math. Forum 3 (2008), 1261-1268.

[26] R. Schmidt, Subgroup lattices of groups, de Gruyter, 1994, Berlin.

[27] M. Tărnăuceanu, Subgroup commutativity degrees of finite groups, J. Algebra 321 (2009), $2508-2520$.

[28] M. Tărnăuceanu, Addendum to "Subgroup commutativity degrees of finite groups", J. Algebra 337 (2011), 363-368.

[29] M. Tărnăuceanu, On the factorization numbers of some finite $p$-groups, Ars Comb., to appear.

[30] J. Zhang, A note on finite groups satisfying permutizer condition, Kexue Tongbao (English Ed.) 31 (1986), 363-365. 


\section{ERRATA CORRIGE - 10TH OF SEPTEMBER 2017}

After the publication of the previous results in:

D.E. Otera and F.G. Russo, Permutability degrees of finite groups, Filomat 30 (2016), 2165-2175.

some errors have been reported to our attention, thanks to the communication of some colleagues (in particular we thank Prof. Paz Jiménez Seral). As noted below, the main results are not directly involved, but we cannot avoid to give more details on some points which might be ambiguous if we don't do it.

(1) Definition of $P(G)$ in Introduction. Two lines below. Replace the sentence "Note that for any $X \in \mathcal{L}(P(G))$ one has $P_{G}(X)=G$ " by "Note that when $X=P(G)$ one has $P_{G}(X)=P(G)$ ".

(2) The value of $p d\left(D_{8}\right)$ in Example 3.2 is wrong. In fact $p d\left(D_{8}\right)=1$, in agreement with Proposition 6.1 when we show that $p d\left(D_{2 p}\right)=1$ for dihedral groups (of order $2 p$ with $p$ odd). The error of Example 3.2 is here:

$$
4=\left|M_{1}\right|=\left|P_{D_{8}}(H)\right|=\left|P_{D_{8}}(K)\right|=\left|P_{D_{8}}(V)\right|=\left|P_{D_{8}}(U)\right|,
$$

in fact we must replace this computation with

$$
8=\left|P_{D_{8}}(H)\right|=\left|P_{D_{8}}(K)\right|=\left|P_{D_{8}}(V)\right|=\left|P_{D_{8}}(U)\right| .
$$

Consequently, $P\left(D_{8}\right)=D_{8}$ and $P\left(D_{8}\right) \neq Q\left(D_{8}\right)$. In particular, the final two sentences from "This example shows ..." until the end of Example 3.2 must be removed. In addition,

(a) the final sentence of Remark 2.3 must be removed;

(b) the sentence just before Theorem 5.1 must be removed;

(c) the sentences before Theorem 5.2 "Of course, $D_{8} \ldots P\left(D_{8}\right) \simeq C_{2}$ " must be removed.

(d) the sentences "On the other hand... further Theorem 5.2" before Theorem 5.3 must be removed.

(3) Proof of the lower bound in Theorem 4.1. There is a misprint: it must be

$$
\sum_{X \in \mathcal{L}(G)}\left|P_{H}(X)\right| \geq \sum_{X \in \mathcal{L}(H)}\left|P_{H}(X)\right|
$$

(4) In Theorem 4.3 we use an assumption, not justified properly in the proof, but this may be motivated by evidences of computational nature. Therefore Theorem 4.3 must be reformulated in the following way:

Theorem 8.1. Let $G$ be a noncyclic group and $p$ the smallest prime divisor of $|G|$. If $P_{G}(X)$ is a proper subgroup of $G$ for all $X \in \mathcal{L}(G),|P(G)|=p$ and $|\mathcal{L}(G)|=m+\frac{p^{m+1}+p-2}{p-1}$ for some $m \geq 0$, then

$$
p d(G) \leq \frac{p^{m+1}+2 p^{2}+(m-3) p-m}{p^{m+2}+(m+1) p^{2}-(m+2) p} .
$$

The proof is that of Theorem 4.3 without the first two sentences. 